\title{
Highly Oriented Carbon Nanotube Supercapacitors
}

Kohei Komatsubara ${ }^{1 \dagger}$, Hiroo Suzuki ${ }^{1{ }^{1 *}}$, Hirotaka Inoue ${ }^{1}$, Misaki Kishibuchi ${ }^{1}$, Shona

Takahashi $^{1}$, Tatsuki Marui ${ }^{1}$, Shigeyuki Umezawa ${ }^{1,2}$, Tomohiro Nakagawa ${ }^{1}$, Kyohei Nasu $^{l}$, Mitsuaki Maetani $^{l}$, Yuichiro Tanaka ${ }^{l}$, Miyato Yamada ${ }^{l}$, Takeshi Nishikawa ${ }^{1}$, Yoshifumi Yamashita ${ }^{1}$,Masaki Hada ${ }^{3}$ Yasuhiko Hayashi ${ }^{{ }^{*}}$

${ }^{1}$ Graduate School of Natural Science and Technology, Okayama University, Okayama 700-8530, Japan.

${ }^{2}$ Seiwa Electric MFG. CO., Ltd., Kyoto 610-0192, Japan

${ }^{3}$ Faculty of Pure and Applied Sciences, Tsukuba Research Center for Energy Materials

Science (TREMS), University of Tsukuba, Tsukuba 305-8573, Japan.

E-mail address: hiroo.suzuki@okayama-u.ac.jp (H. Suzuki).

E-mail address: hayashi.yasuhiko@okayama-u.ac.jp (Y. Hayashi). 


\section{Supplementary note 1: Energy density and power density of CNT-SCs.}

We calculated the energy density $(E)$ and power density $(P)$ of CNT-SCs. $E$ and $P$ were calculated using the following equations

$$
\begin{gathered}
E=\frac{C\left(V-V_{d}\right)^{2}}{2} \\
P=\frac{E}{\Delta t}
\end{gathered}
$$

where $C$ is specific capacitance, $V$ is the voltage window, $V_{\mathrm{d}}$ is the initial voltage drop, and $\Delta t$ is the discharge time. We obtained the values of $E$ and $P$ for the $0^{\circ}-, 45^{\circ}-$, and $90^{\circ}$-CNT-SC as shown in Table S1.
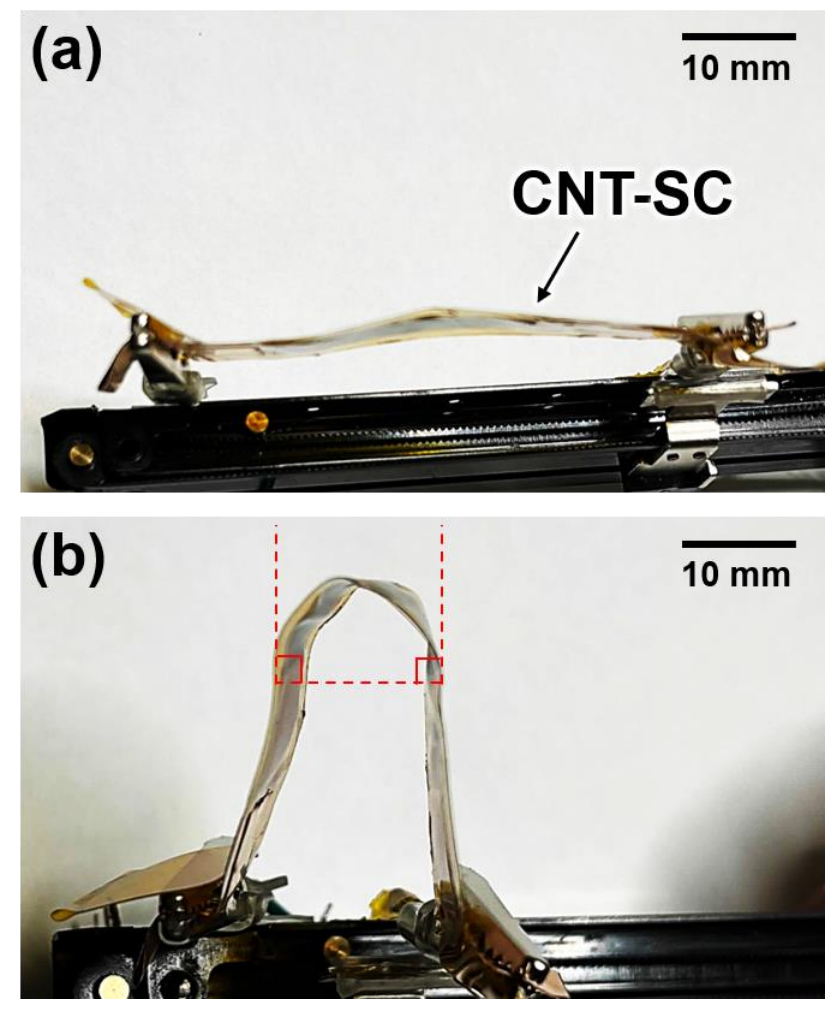

Fig. S1: Pictures of bending tests of CNT-SCs. (a) Unbent and (b) bent CNT-SC. 
(a)

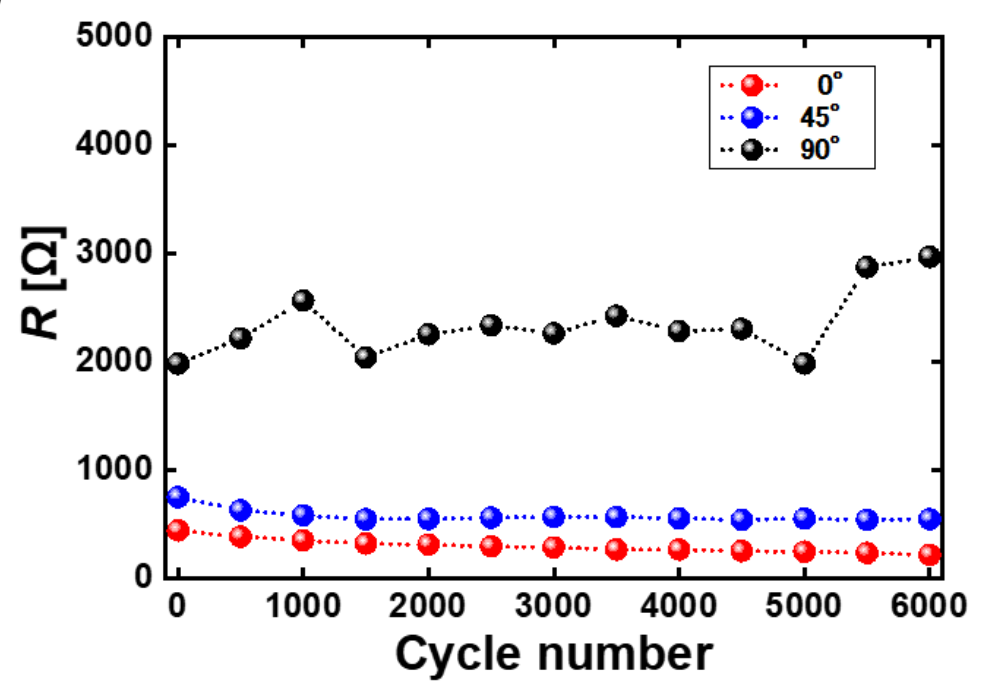

(b)

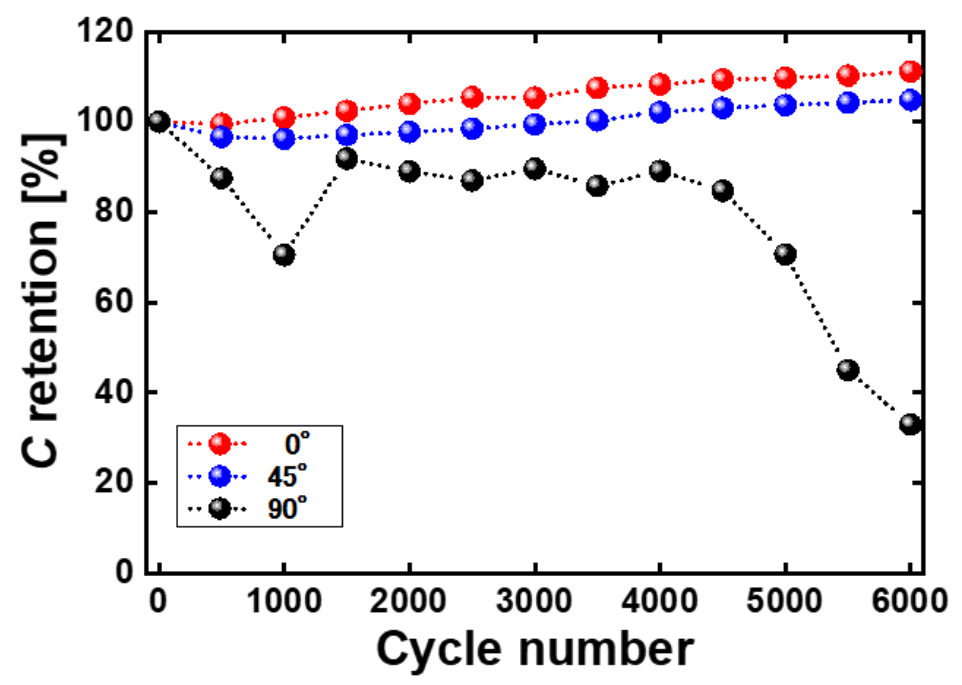

Fig. S2: Cyclic charge-discharge robustness of CNT-SCs. (a) $R$ and (b) $C$ retention values of CNT-SCs. 
Table S1: Energy density and power density of CNT-SCs.

\begin{tabular}{|c|c|c|c|c|}
\hline & \multicolumn{2}{|c|}{ Flat } & \multicolumn{2}{c|}{ Bending } \\
\hline$\theta\left[{ }^{\circ}\right]$ & $E[\mathrm{Wh} / \mathrm{kg}]$ & $P[\mathrm{~W} / \mathrm{kg}]$ & $E[\mathrm{Wh} / \mathrm{kg}]$ & $P[\mathrm{~W} / \mathrm{kg}]$ \\
\hline 0 & 10.4 & 1186 & 10.8 & 1172 \\
\hline 45 & 7.2 & 1102 & 7.5 & 1137 \\
\hline 90 & 3.2 & 931 & 3.4 & 999 \\
\hline
\end{tabular}

Table S2: Fitting parameters of CNT-SCs calculated with equivalent circuits.

\begin{tabular}{|c|c|c|c|}
\hline & $0^{\circ}$ & $45^{\circ}$ & $90^{\circ}$ \\
\hline$R_{1}[\Omega]$ & 27.0 & 161.0 & 498 \\
\hline$R_{2}[\Omega]$ & 6.4 & 91.6 & 106.5 \\
\hline $\mathrm{CPE}_{2}-\mathrm{T}$ & $3.0 \mathrm{E}-7$ & $5.4 \mathrm{E}-7$ & $5.6 \mathrm{E}-7$ \\
\hline $\mathrm{CPE}_{2}-\mathrm{P}$ & 1.0 & 0.9 & 0.8 \\
\hline$R_{3}[\Omega]$ & - & 116.8 & - \\
\hline $\mathrm{CPE}-\mathrm{T}$ & - & $9.0 \mathrm{E}-5$ & - \\
\hline $\mathrm{CPE}-\mathrm{P}$ & - & 0.9 & 45.5 \\
\hline$W o-\mathrm{R}$ & 2.4 & 0.6 & $4.5 \mathrm{E}-2$ \\
\hline$W o-\mathrm{T}$ & $3.4 \mathrm{E}-3$ & $2.1 \mathrm{E}-4$ & 0.3 \\
\hline Wo-P & 0.4 & 0.4 & - \\
\hline
\end{tabular}

